\title{
ac response of an atomic tunnel junction
}

\author{
Wei Zheng, Yadong Wei, and Jian Wang \\ Department of Physics, The University of Hong Kong, Pokfulam Road, Hong Kong, China
}

Hong Guo

Centre for the Physics of Materials and Department of Physics, McGill University, Montreal, PQ, Canada H3A 2T8

(Received 24 November 1999; revised manuscript received 8 February 2000)

\begin{abstract}
By combining density functional analysis with the solution of a three-dimensional quantum scattering problem, and applying appropriate ac transport theory, we investigated the ac transport response of Si atomic tunnel junctions in the resonance tunneling regime. Our results show that transmission channels of both the leads and the atomic section contribute to the dynamic conductance. The ac response is found to be determined by the average channel transmission weighted by the corresponding density of states, explaining a counterintuitive result that at resonance tunneling the ac response may be capacitivelike. As the ac frequency is increased, the resonance peak of dynamic conductance can be split into two, a phenomenon that can be explained analytically.
\end{abstract}

\section{INTRODUCTION}

Through a number of experimental techniques such as scanning probe microscopy ${ }^{1}$ (SPM) and break junctions, ${ }^{2}$ it is now possible to measure the conductance of atomic junctions consisting of a single atom or several atoms. Atomic junctions can be realized by pulling one or several atoms out of a metal surface using a SPM tip, ${ }^{3}$ or by adsorbing foreign atoms on the tip ${ }^{1}$ and flowing a current through them. On the other hand, in the mechanically controllable break junction technique, ${ }^{4-6,2}$ a piece of metal is bent until it is broken. The clean fracture surfaces are then brought back to form atomic size metallic contacts. Results for conductance quantization, ${ }^{5}$ shot noise, ${ }^{7}$ and transition between vacuum tunneling and contact for different metals ${ }^{5}$ have been reported using break junctions. Finally, a tunneling transistor incorporating single, nanometer-scale $\mathrm{Al}$ particles with a gate electrode has been developed, ${ }^{8}$ which can be used to measure the spectrum of discrete electronic states and the gate-voltage dependence of the resonance level widths.

Experimental studies of atomic wires and tunnel junctions have motivated several theoretical investigations of these systems. Lang $^{9}$ has calculated the resistance of atomic wires by solving the Lippmann-Schwinger equation in the framework of density functional theory and predicted negative differential resistance for the tunneling regime. ${ }^{10}$ Good consistency between theoretical and experimental results for $\mathrm{Xe}$ atomic junctions was reported. ${ }^{1}$ For $\mathrm{Na}$ and $\mathrm{C}$ atomic wires, anomalous behavior of the dc conductance was found and believed to be a result of atomic orbitals. ${ }^{11,12}$ Wan et al. ${ }^{13}$ and Mozos et al. ${ }^{14}$ have calculated the dc conductance of $\mathrm{Al}$ and $\mathrm{Si}$ atomic wires by combining a pseudopotential method and three-dimensional (3D) quantum scattering theory, and reported conductance quantization for wires consisting of two or more atoms. Si atomic wires were found to be metallic even though bulk $\mathrm{Si}$ is a semiconductor, a fact that was confirmed by the 1D band structure of an infinitely long $\mathrm{Si}$ chain. ${ }^{14}$ The dc conductance of atomic wires has also been inferred from the density of states (DOS), ${ }^{15}$ or by direct evaluation of the Kubo formula using Kohn-Sham eigenstates. ${ }^{16}$ Using a self-consistent tight-binding model, Cuevas et al. ${ }^{17}$ have studied the evolution of conducting channels in atomic contacts under elastic deformation. For a linear chain of $\mathrm{Au}$ atoms suspended between two electrodes, a Peierls-like transition was found as the average bond length increased. $^{18}$

Another important problem, which is the focus of this paper, concerns the ac transport response of atomic junctions. Recently, dynamic transport properties of atomic wires have been investigated using a first-principles pseudopotential method, from which the low frequency dynamic conductance of $\mathrm{Al}$ atomic wires and the electrochemical capacitance of $\mathrm{Al}$ and $\mathrm{Si}$ wires were calculated. ${ }^{19,20}$ For the transmissive Al wire, a large inductive ac response was obtained. The dynamic conductance as a function of the scattering electron energy showed a spiky behavior, and this behavior became sharper as the wire length was increased. For atomic scale capacitors, quantum effects become important due to the finite density of states and tunneling effects. ${ }^{21,20}$ In contrast to the classical situation, the quantum capacitance increases with the distance $d$ between the two capacitor plates at small $d .^{20,22}$ While useful information has been obtained concerning the ac transport response of atomic scale wires, so far all studies were in the limit of very small ac frequency $\omega$, i.e., in the $\omega \rightarrow 0$ limit. When the external ac bias has such small frequencies, the system can be assumed to be in or near equilibrium to allow the application of well established linear response theory. However, the $\omega \rightarrow 0$ limit may not capture the entire physical picture of dynamic quantum transport since atomic devices can operate at a finite frequency. At a simpler level, it is also interesting to know the transport coefficients at the order $\omega^{2}$ and higher. Clearly, it is highly desirable to investigate quantum transport properties under the condition of finite ac frequency, and it is the purpose of this paper to fulfill this goal.

In order to investigate quantum transport under a timevarying bias, the induced displacement current must be included as a result of charge accumulation in the system. An- 
other important physical requirement for transport theory is gauge invariance, which means that the transport properties depend only on the voltage difference. ${ }^{23}$ For theoretical studies of the ac response of atomic junctions, satisfying these physical requirements is crucial due to the small DOSs of the junctions. Recently, we have developed a theoretical formalism based on nonequilibrium Green's functions (NEGFs) which satisfies these physical requirements ${ }^{24}$ and is applicable at a finite ac frequency. ${ }^{25,26}$ Our analysis of the dynamic conductance of a $\mathrm{Si}$ atomic tunnel junction is based on this theory (see below). In particular, we consider an atomic junction formed by a single $\mathrm{Si}$ atom sandwiched between two metallic leads in the tunneling regime, for which a resonant tunneling behavior can be obtained. Our results show that transmission channels of both the leads and the scattering region contribute to the dynamic conductance. The ac response is found to be determined by the average channel transmission coefficient weighted by the appropriate density of states, explaining a counterintuitive result that at resonant tunneling one may obtain a capacitivelike ac response. Finally, our numerical data can be qualitatively understood from analytical approaches near resonance points; hence, an overall physical picture emerges of ac quantum transport through atomic tunnel junctions in the resonance tunneling regime.

\section{ANALYSIS, RESULTS, AND DISCUSSION}

Within the NEGF formalism the following dynamic conductance $G_{\alpha \beta}(\omega)$, measured from a lead labeled $\alpha$ to one labeled $\beta$, was derived: ${ }^{24}$

$$
G_{\alpha \beta}=G_{\alpha \beta}^{c}-G_{\beta}^{d} \frac{\sum_{\gamma} G_{\alpha \gamma}^{c}}{\sum_{\gamma} G_{\gamma}^{d}} .
$$

In this expression, the quantity with superscript $d$ is the conductance coefficient contributed by the displacement current, while that with superscript $c$ is contributed by the particle current. They are given by ${ }^{27}$

$$
G_{\beta}^{d}(\omega)=-q \omega \int \frac{d E}{2 \pi} \operatorname{Tr}\left(\bar{g}_{\beta}^{<}\right)
$$

and

$$
G_{\alpha \beta}^{c}(\omega)=-q \int \frac{d E}{2 \pi} \operatorname{Tr}\left[i \bar{g}_{\beta}^{<} \Gamma_{\alpha}+\left(\bar{G}_{0}^{r}-G_{0}^{a}\right) \bar{\sigma}_{\alpha}^{<} \delta_{\alpha \beta}\right],
$$

where $q$ is the electron charge. Before defining the rest of the quantities in these equations, we emphasize that inclusion of the displacement current contribution preserves current conservation under ac conditions, and the expression (1) also satisfies gauge invariance. These facts are mathematically expressed $^{28}$ as $\Sigma_{\alpha} G_{\alpha \beta}=\Sigma_{\beta} G_{\alpha \beta}=0$. In the above equations, $g \equiv g(E, E)$ and $\bar{g} \equiv \bar{g}(E+\hbar \omega, E)$ : they are double-time Fourier transforms of the Green's function $g\left(t, t^{\prime}\right)$. The Green's functions in Eqs. (2) and (3) are given by ${ }^{29,30,24}$

$$
\bar{g}_{\alpha}^{<}=\bar{G}_{0}^{r} \bar{\sigma}_{\alpha}^{<} G_{0}^{a},
$$

$$
\bar{\sigma}_{\alpha}^{<}=\frac{i q}{\omega} \Gamma_{\alpha}(f-\bar{f}),
$$

where $f$ is the Fermi-Dirac distribution. $G_{0}^{r}$ and $G_{0}^{a}$ are equilibrium Green's functions and are related to the scattering matrix through the Fisher-Lee relation. ${ }^{31}$ In the language of scattering matrix theory, ${ }^{32}$ the conductance due to the particle current is ${ }^{30}$

$$
\begin{aligned}
G_{\alpha \beta}^{c}(\omega)= & q^{2} \int \frac{d E}{2 \pi} \operatorname{Tr}\left[1_{\alpha} \delta_{\alpha \beta}-S_{\alpha \beta}^{\dagger}(E) S_{\alpha \beta}(E+\hbar \omega)\right] \\
& \times \frac{f(E)-f(E+\hbar \omega)}{\hbar \omega},
\end{aligned}
$$

which is the same as that given by Büttiker et al. ${ }^{32}$

Near a quantum resonance, the scattering matrix is described by the Breit-Wigner formula

$$
S_{\alpha \beta}=\delta_{\alpha \beta}-i \frac{\Gamma / 2}{\Delta E+i \Gamma / 2},
$$

where $\Delta E=E-E_{0}$ and we have assumed that the system is symmetric (see below). For this particular scattering matrix the dynamic conductance can be obtained exactly, ${ }^{33,24}$

$$
\operatorname{Re} G_{11}=\frac{\Gamma}{8 \pi \omega}\left[\arctan \left(\frac{\Delta E+\omega}{\Gamma / 2}\right)-\arctan \left(\frac{\Delta E-\omega}{\Gamma / 2}\right)\right]
$$

and

$$
\begin{aligned}
\operatorname{Im} G_{11}= & \frac{\Gamma}{16 \pi \omega} \ln \left\{\left[(\Delta E+\omega)^{2}+(\Gamma / 2)^{2}\right]\right. \\
& \left.\times\left[(\Delta E-\omega)^{2}+(\Gamma / 2)^{2}\right]\left[(\Delta E)^{2}+(\Gamma / 2)^{2}\right]^{-2}\right\} .
\end{aligned}
$$

The dynamic conductance $G=G_{11}$ is a complex quantity, with its real part $G_{R}$ describing dissipation and its imaginary part $G_{I}$ characterizing the phase difference between the current and the voltage. At small frequency, it is appropriate to describe $G(\omega)$ using Buttiker's formalism, ${ }^{28}$ according to which the dynamic conductance can be expanded as a power series in frequency $\omega, G_{\alpha \beta}(\omega)=G_{\alpha \beta}(0)-i \omega E_{\alpha \beta}+O\left(\omega^{2}\right)$. $E_{\alpha \beta}$ is the emittance defined as

$$
E_{\alpha \beta}=\frac{d N_{\alpha \beta}}{d E}-D_{\alpha \beta}
$$

where $D_{\alpha \beta}$ can be expressed in terms of the local density of states $d n_{\alpha} / d E$ in the Thomas-Fermi approximation

$$
D_{\alpha \beta}=\int d^{3} r \frac{\left[d n_{\alpha}(r) / d E\right]\left[d n_{\beta}(r) / d E\right]}{d n(r) / d E} .
$$

Here $E_{\alpha \beta}$ satisfy the current conservation and gauge invariant condition $\Sigma_{\alpha} E_{\alpha \beta}=\Sigma_{\beta} E_{\alpha \beta}=0$. In Eq. (10), $d N_{\alpha \beta} / d E$ is the global partial DOS,

$$
\frac{d N_{\alpha \beta}}{d E}=\frac{1}{4 \pi i}\left(S_{\alpha \beta}^{\dagger} \frac{d S_{\alpha \beta}}{d E}-\frac{d S_{\alpha \beta}^{\dagger}}{d E} S_{\alpha \beta}\right),
$$

where $d N_{\alpha} / d E=\Sigma_{\beta} d N_{\alpha \beta} / d E$ is the injectivity. Two different behaviors can be observed depending on the sign of the 
emittance $E_{11}$ (or the sign of $G_{I}$ ). For single-channel transmission, if $E_{11}$ is positive then the capacitivelike behavior dominates; otherwise, the system behaves like an inductor. This can be understood using scattering matrix theory. ${ }^{28}$ For a capacitor, the transmission coefficient is zero, and hence $d N_{12} / d E=0$ from Eq. (12). As a result, $E_{12}$ is negative. Since $E_{11}+E_{12}=0$ from current conservation, $E_{11}$ is positive for a capacitor. For an inductor, $S_{11}=0$, i.e., complete transmission. From Eq. (10), $E_{11}$ is negative. In summary, for single-channel transmission, complete transmission corresponds to an inductivelike behavior and complete reflection corresponds to a capacitivelike behavior.

The numerical calculation of expression (1) proceeds as follows. ${ }^{13}$ First, we determine the equilibrium properties of the open system, the atom and the two long leads, using an $a b$ initio pseudopotential calculation. By solving the KohnSham equation self-consistently, we obtain the Kohn-Sham wave function and the self-consistent effective potential $V_{e f f}(\mathbf{r}) \equiv \delta U / \delta \rho(\mathbf{r})$, where $U[\rho]$ is the total self-consistent potential energy and $\rho$ is the electron density. We have used the parametrization of Ref. 34 for the exchange-correlation term, and a local pseudopotential ${ }^{35}$ for the core. We emphasize that in calculating $V_{\text {eff }}$ one has to make sure that the lead is long enough to approach the asymptotic limit, such that the eigenstates obtained from the equilibrium density functional calculation can be brought into the form of scattering states of the open system. ${ }^{36,37}$ Given the effective potential, the single-electron scattering problem is solved ${ }^{38}$ and the dynamic conductance is finally obtained using Eqs. (1) and (3).

In this work we model the leads with the jellium model $^{9,13,39}$ where positive charges are uniformly distributed with the radius of a free electron sphere $r_{s}=2.0$ mimicking high density metallic electrodes. The leads have a crosssectional area of $8.11 \times 8.11$ (a.u.) ${ }^{2}$ and length $L=30.78$ a.u. The supercell volume used in our calculation is 25.39 $\times 25.39 \times 2(L+d)$ a.u. ${ }^{3}$, where $d$ is the distance between the $\mathrm{Si}$ atom and the jellium electrode. We choose $d=8.0$ a.u., which is much larger than $d=2.3$ a.u., the latter being the equilibrium distance of a $\mathrm{Si}$ atom on top of a jellium plane. ${ }^{40}$ This creates two vacuum barriers between the $\mathrm{Si}$ atom and the two electrodes. There are a total of 120 electrons in the supercell and we have used an energy cutoff of $8 \mathrm{Ry}$ in the plane wave analysis of $V_{\text {eff }}$.

Figure 1 plots the total transmission coefficient as a function of the Fermi energy. We observe that there are three resonant peaks at $E_{1}=-0.3632$ a.u., $E_{2}=-0.1165$ a.u., and $E_{3}=-0.1056$ a.u. For the small resonant energy $E_{F}=E_{1}$, there is only one transmission channel and at $E_{1}$ the reflection coefficient is zero. For larger energies $E_{2}$ and $E_{3}$, there are six transmission channels in the lead; hence the total reflection coefficients at $E_{2}$ and $E_{3}$ are nonzero. At $E_{2}$ the conductance is through the first channel with conductance $G=1$, while at $E_{3}$ the conductance is through the second and third channels with $G=2$. Note that the resonant peak at $E_{3}$ is much sharper than that at $E_{2}$, indicating a much longer dwell time or much larger density of states. We estimate $\Gamma$ $\sim 0.0056$ and 0.0008 at $E_{2}$ and $E_{3}$, respectively, using the Breit-Wigner formula.

Figure 2 depicts the dynamic conductance $G$ versus Fermi energy at small frequency $\omega=0.0006$ a.u. (solid line for $G_{R}$

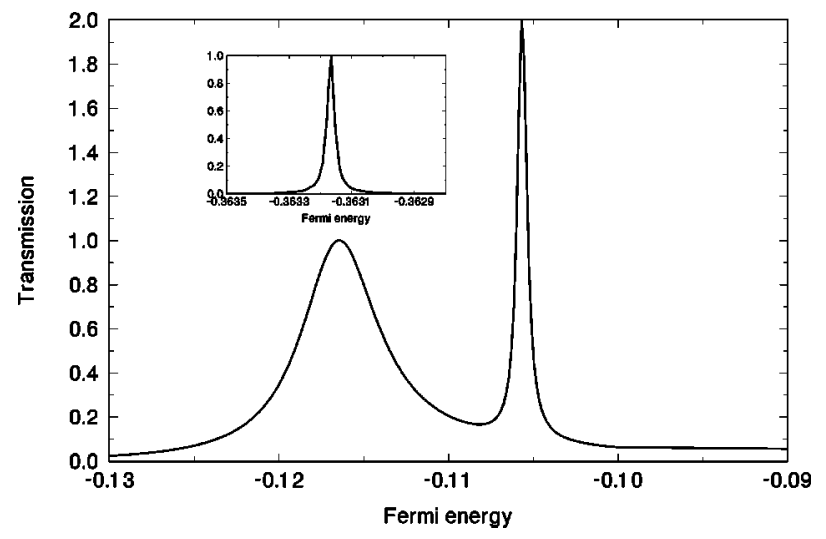

FIG. 1. Transmission coefficient versus the Fermi energy, showing resonant peaks at $E_{2}=-0.1165$ a.u. and $E_{3}=-0.1056$ a.u. Inset: transmission coefficient versus energy for the resonant peak at $E_{1}$. The sharp peaks indicate resonance tunneling mediated by the atomic orbitals.

and dotted line for $\left.G_{I}\right) .{ }^{41}$ Several observations are in order. (1) When the frequency is kept small, e.g., $\omega=0.0006$ a.u., the dc behavior is still maintained in the real part of the dynamic conductance. However, there is a large decrease of the peak height near $E_{F}=E_{3}$. (2) An additional big peak emerges near $E=-0.046$ a.u., which is at the threshold of the eighth subband. This extra peak can be explained as follows: when approaching the eighth subband threshold two additional conducting channels are available, resulting in a sharp peak in the DOS. This gives rise to a peak in both $G_{R}$ and $G_{I}$ near the subband threshold. (3) From $G_{I}$ versus $E_{F}$, we observe that the ac response shows a capacitivelike behavior (negative $G_{I}$ ) except near resonances $E_{F}=E_{1}$ (not shown in the figure) and $E_{F}=E_{3}$; at the latter energies inductivelike behaviors are observed. However, at $E_{F}=E_{2}$, a resonant point, the system responds capacitively.

The response behavior at $E_{F}=E_{1}$ is the typical inductivelike behavior for complete transmission and is well understood ${ }^{24}$ since at $E_{1}$ there is only one transmission channel. However, what is the reason that the ac response is so different for $E_{2}$ and $E_{3}$ although both energies give resonant dc transmission? To understand the interesting difference we rewrite Eq. (10) as follows in the discrete potential approximation: ${ }^{21}$

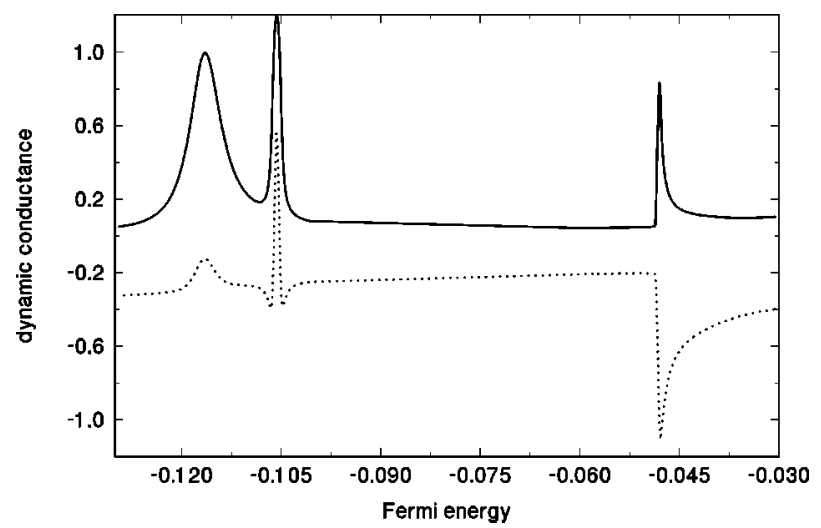

FIG. 2. The dynamic conductance versus energy at $\omega=0.0006$. Solid line: the real part $G_{R}$; dotted line: the imaginary part $G_{I}$. 


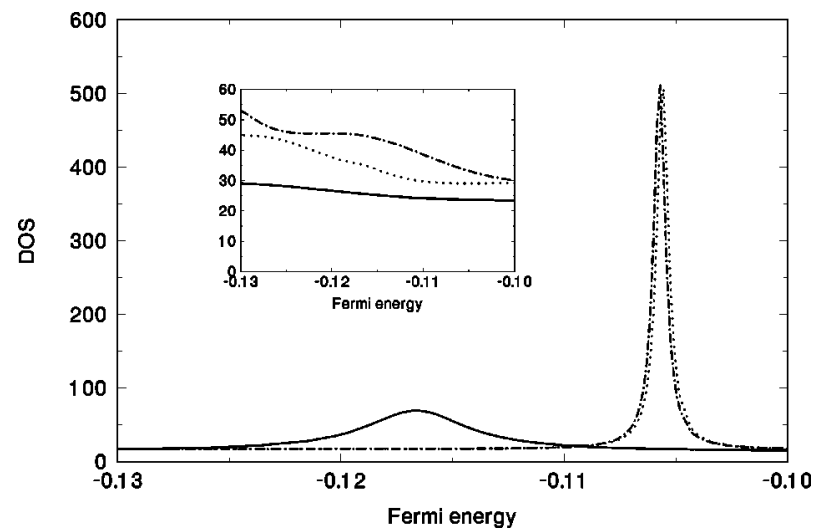

FIG. 3. Density of states $d N_{n} / d E$ versus energy at $\omega=0.0006$ for the first three transmission channels. Solid line: $n=1$; dotted line: $n=2$; dash-dotted line: $n=3$. Clearly, at $E_{2}=-0.1165$ a.u. only one channel has a large DOS while at $E_{3}=-0.1056$ a.u. there are two channels having very large DOSs. Inset: DOS of the last three channels, solid line: $n=4$; dotted line: $n=5$; dash-dotted line: $n=6$.

$$
E_{11}=\frac{d N_{11}}{d E}-\frac{\left(d N_{1} / d E\right)\left(d N_{1} / d E\right)}{d N / d E} .
$$

Since for a symmetric system $d N / d E=2 d N_{1} / d E$, and $d N_{12} / d E=(T / 2) d N / d E$ for a single channel, ${ }^{21}$ we have $d N_{11} / d E=d N_{1} / d E-d N_{12} / d E=(1-T) / 2(d N / d E)$. So for the multichannel case we have

$$
E_{11}=\frac{d N_{11}}{d E}-\frac{1}{4} \frac{d N}{d E}=-\frac{1}{4}\left(2 T_{a v}-1\right) \frac{d N}{d E},
$$

where the average transmission coefficient weighted by the DOS, $T_{a v}$, is defined as ${ }^{21}$

$$
T_{a v}=\frac{\sum_{n} T_{n} d N_{n} / d E}{d N / d E} .
$$

Here $T_{n}$ is the transmission probability for channel $n$ and $d N_{n} / d E$ is the corresponding DOS, which is proportional to the dwell time in that channel. Equation (14) indicates that for a multichannel scattering process, the average transmission coefficient determines the ac response of the system: if $T_{a v}>1 / 2$ it is inductivelike, otherwise it is capacitivelike. For $E_{F}=E_{2}$, the electron resonantly tunnels via the first channel and is reflected back for the other channels: $T_{1}=1$ while $T_{2,3, \ldots, 6}=0$. Since resonant tunneling is characterized by a long dwell time, ${ }^{42}$ the dwell time or DOS of the first channel is therefore larger than that of the other channels. This conclusion is indeed consistent with our numerical data: at $E_{2}, \quad d N_{i} / d E=70,18,18,25,35,45$ for channels $i$ $=1,2, \ldots, 6$, as plotted in Fig. 3. Therefore, according to Eq. (15) we obtain $T_{a v} \sim 0.35$ or $E_{11}>0$ at $E_{2}$, resulting in a capacitivelike behavior rather than the inductivelike behavior usually expected at resonances. For $E_{F}=E_{3}$, the electron tunnels through the second and the third channels, i.e., $T_{2}$ $=T_{3}=1$. Because of the much sharper resonance at $E_{3}$, the DOSs $d N_{2} / d E$ and $d N_{3} / d E$ are much larger than the rest: $d N_{i} / d E=18,495,425,24,29,33$ (see Fig. 3). According to Eq. (15) a $T_{a v}$ of order 1 is expected. Indeed, our numerical
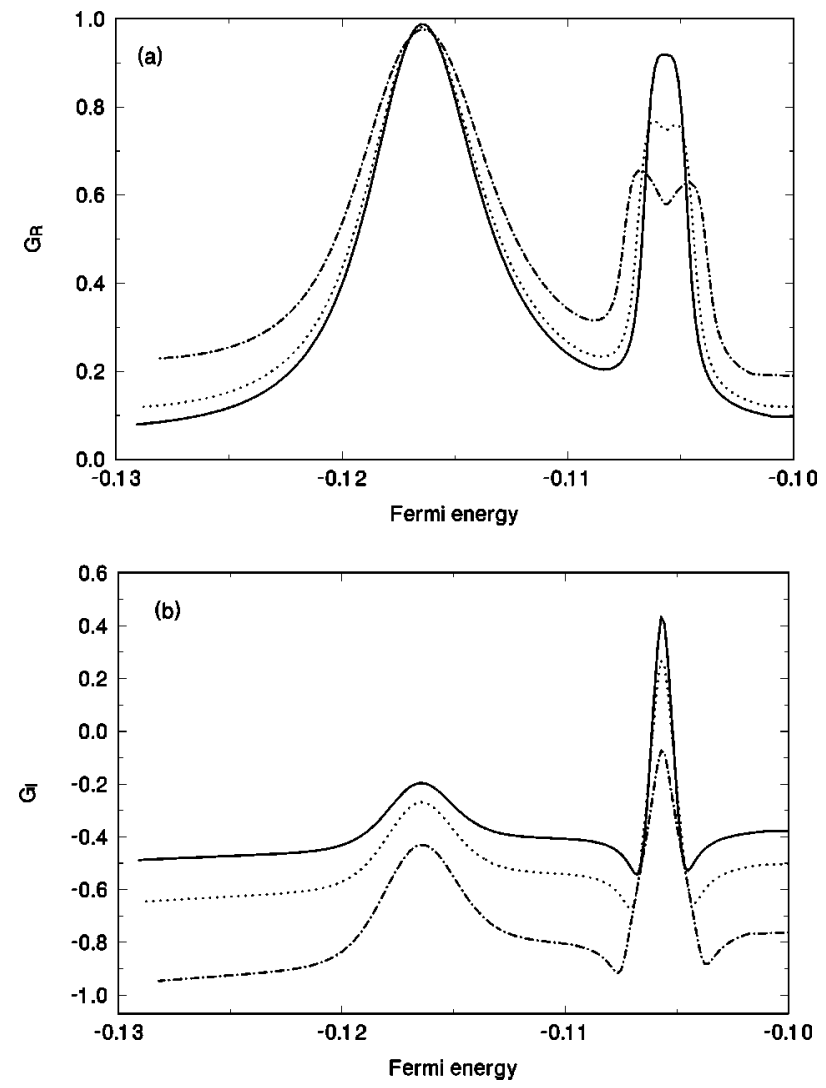

FIG. 4. Dynamic conductance $G(\omega)$ versus energy for different frequencies. (a) The real part of $G(\omega), G_{R}$; (b) the imaginary part $G_{I}$. Solid line: $\omega=0.0009$; dotted line: $\omega=0.0012$; dash-dotted line: $\omega=0.0018$.

date gave $T_{a v} \sim 0.9$, leading to $E_{11}<0$ and hence an inductivelike response at $E_{3}$. These results clearly demonstrate that the ac response is directly dependent on the transmission distribution among the channels.

As mentioned above, there is a large reduction of peak height at $E_{3}$ at a finite frequency (see Fig. 2). This behavior can be understood as follows. For small $\omega$ and near a resonance, we can expand the Breit-Wigner formula to obtain $G(\omega)=G(0)-i \omega E_{11}+\omega^{2} K_{11}$, where $K_{11}$ is given by ${ }^{33}$

$$
K_{11}=\frac{\pi^{2}}{\Gamma}\left[(\Delta E)^{2}-\Gamma^{2} / 12\right]\left(\frac{d N}{d E}\right)^{3}
$$

with

$$
\frac{d N}{d E}=\frac{1}{2 \pi} \frac{\Gamma}{(\Delta E)^{2}+(\Gamma / 2)^{2}} .
$$

This allows us to conclude that at resonance $G_{R}(\omega)$ $<G_{R}(0)$ because $K_{11}<0$. Thus, in general, at a quantum resonance the dynamic conductance is smaller than the corresponding dc conductance. Quantitatively, for $E_{F}=E_{2}$ the DOS is numerically not very large, and hence $K_{11}$ is numerically negligible when multiplied by $\omega^{2}$. As a result, the decrease of $G(\omega)$ (see Fig. 2) is only a few percent at $E_{2}$. On the other hand, the DOS at $E_{3}$ is much larger, leading to a significant reduction of $G(\omega)$.

From the above discussion, we expect the transmission peak reduction to increase as $\omega$ is increased. Figure 4(a) 
showsthis behavior, which is especially clear at $E_{3}$, for three different frequencies. We observe that as $\omega$ increases the peak of $G_{R}$ changes slightly at $E_{2}$ but decreases drastically at $E_{3}$. In addition, for higher frequencies the peak of $G_{R}$ splits in two. From Eq. (16), if $\Delta E> \pm \Gamma / \sqrt{12}, K_{11}>0$. Thus as the energy is varied to cross the resonance point, $K_{11}$ changes from positive to negative to positive, explaining why the peak is broadened and can be split into two. When the frequency is increased, both the inductivelike and the capacitivelike behaviors of the dynamic conductance $G_{I}$ are enhanced except around $E_{3}$. This is reasonable for small frequency since $G_{I}$ varies linearly with $\omega$. When frequency is not very small, we examine Eq. (9), from which we see that the peak of $G_{I}$ at resonance will increase with $\omega$ if $\Gamma \gg \omega$ and decrease for $\Gamma<\omega$. Indeed, this is what we see from Fig. 4(b). The peak of $G_{I}$ increases near $E_{2}(\Gamma \sim 0.0056)$, but decreases slightly near $E_{3} \quad(\Gamma \sim 0.0008)$. We also note that the overall curve of $G_{I}$ is shifted downward and eventually the response near $E_{3}$ will be capacitivelike. This is due to the fact that other reflection channels respond capacitively.

\section{SUMMARY}

Our analysis shows that atomic tunnel junctions can have a very rich ac transport response due to contributions of channels both in the leads and through the atom. For ex- ample, for the Si junction studied here there are six channels in the leads but only one can go through at $E_{2}$ and two can go through at $E_{3}$. But all the channels contribute to the ac response through the average transmission coefficients, leading to totally different responses at $E_{2}$ and $E_{3}$. In particular, this explains the counterintuitive result that at a quantum resonance transmission one may observe a capacitive ac response. In this work we used vacuum barriers to form the atomic tunnel junction; experimentally one may be able to establish junctions using insulating materials to isolate an atomic sized cluster, as was already done in another context. ${ }^{8}$ We expect a qualitatively similar ac response for these more complicated systems in the resonance tunneling regime. Certainly, our predictions point to interesting dynamic responses of atomic scale tunnel junctions for further experimental study.

\section{ACKNOWLEDGMENTS}

We gratefully acknowledge financial support by a RGC grant from the SAR Government of Hong Kong under Grant No. HKU 7115/98P and a CRCG grant from the University of Hong Kong. H.G. is supported by NSERC of Canada and FCAR of Québec. We thank the Computer Center of The University of Hong Kong for computer facilities.
${ }^{1}$ A. Yazadani, D.M. Eigler, and N.D. Lang, Science 272, 1921 (1996).

${ }^{2}$ E. Scheer et al., Phys. Rev. Lett. 78, 3535 (1997); Nature (London) 394, 6689 (1998).

${ }^{3}$ A.I. Yanson et al., Nature (London) 394, 783 (1998); H. Ohnishi, Y. Kondo, and K. Takayanagi, ibid. 395, 780 (1998).

${ }^{4}$ C.J. Muller, J.M. van Ruitenbeek, and L.J. de Jongh, Phys. Rev. Lett. 69, 140 (1992).

${ }^{5}$ J.M. Krans et al., Phys. Rev. B 48, 14721 (1993); J.M. Krans et al., Nature (London) 375, 767 (1995).

${ }^{6}$ J.L. Costa-Kramer, N. Garcia, P. Garcia-Mochales, and P.A. Serena, Surf. Sci. 342, 1144 (1995).

${ }^{7}$ H.E. van den Brom and J.M. van Ruitenbeek, Phys. Rev. Lett. 82, 1526 (1999).

${ }^{8}$ D.C. Ralph, C.T. Black, and M. Tinkham, Phys. Rev. Lett. 78, 4087 (1997).

${ }^{9}$ N.D. Lang, Phys. Rev. B 52, 5335 (1995).

${ }^{10}$ N.D. Lang, Phys. Rev. B 55, 9364 (1997).

${ }^{11}$ N.D. Lang, Phys. Rev. Lett. 79, 1357 (1997).

${ }^{12}$ N.D. Lang and Ph. Avouris, Phys. Rev. Lett. 81, 3515 (1998).

${ }^{13}$ C.C. Wan et al., Appl. Phys. Lett. 71, 419 (1997).

${ }^{14}$ J.L. Mozos et al., Phys. Rev. B 56, R4351 (1997); J. Phys.: Condens. Matter 10, 2663 (1998).

${ }^{15}$ H. Mehrez, S. Ciraci, A. Buldum, and I.P. Batra, Phys. Rev. B 55, R1981 (1997).

${ }^{16}$ R.N. Barnett and U. Landman, Nature (London) 387, 788 (1997).

${ }^{17}$ J.C. Cuevas, A.L. Yeyati, and A. Martn-Rodero, Phys. Rev. Lett. 80, 1066 (1998); J.C. Cuevas et al., ibid. 81, 2990 (1998).

${ }^{18}$ M. Okamoto and K. Takayanagi, Phys. Rev. B 60, 7808 (1999).

${ }^{19}$ C.C. Wan et al., Phys. Rev. B 55, R13 393 (1997).
${ }^{20}$ J. Wang et al., Phys. Rev. Lett. 80, 4277 (1998).

${ }^{21}$ T. Christen and M. Büttiker, Phys. Rev. Lett. 77, 143 (1996).

${ }^{22}$ X.A. Zhao, J. Wang, and H. Guo, Phys. Rev. B 60, 16730 (1999).

${ }^{23}$ For discussions on gauge invariance, see M. Büttiker and T. Christen, in Quantum Transport in Semiconductor Submicron Structures, edited by B. Kramer (Kluwer, Dordrecht, 1996), p. 263; Z.S. Ma, J. Wang, and H. Guo, Phys. Rev. B 57, 9108 (1998); 59, 7575 (1999).

${ }^{24}$ B.G. Wang, J. Wang, and H. Guo, Phys. Rev. Lett. 82, 398 (1999).

${ }^{25}$ M.K. Yip, W.C. Kwok, J. Wang, and H. Guo (unpublished).

${ }^{26}$ C. Roland, M.B. Nardelli, J. Wang, and H. Guo, Phys. Rev. Lett. 84, 2921 (2000).

${ }^{27}$ To simplify the calculation, we have neglected in Eq. (3) quantities such as $\sigma_{\alpha}^{r, a}$ and $\bar{g}_{\alpha}^{r, a}$, which are correction terms to equilibrium quantities $\Sigma_{0 \alpha}^{r, a}$ and $\bar{G}_{0}^{r, a}$.

${ }^{28}$ M. Büttiker, J. Phys.: Condens. Matter 5, 9361 (1993).

${ }^{29}$ A.P. Jauho, N.S. Wingreen, and Y. Meir, Phys. Rev. B 50, 5528 (1994).

${ }^{30}$ M.P. Anantram and S. Datta, Phys. Rev. B 51, 7632 (1995).

${ }^{31}$ D.S. Fisher and P.A. Lee, Phys. Rev. B 23, 6851 (1981).

${ }^{32}$ M. Büttiker, A. Prêtre, and H. Thomas, Phys. Rev. Lett. 70, 4114 (1993).

${ }^{33}$ M. Buttiker and T. Christen, in Mesoscopic Electron Transport, edited by L.L. Sohn et al. (Kluwer, Dordrecht, 1997), p. 259.

${ }^{34}$ S. Goedecker, M. Teter, and J. Hutter, Phys. Rev. B 54, 1703 (1996).

${ }^{35}$ J. Ihm and M.L. Cohen, Solid State Commun. 29, 711 (1979).

${ }^{36}$ J. Wang, Y.J. Wang, and H. Guo, J. Appl. Phys. 75, 2721 (1994); 
Y.J. Wang, J. Wang, H. Guo, and E. Zaremba, Phys. Rev. B 52, 2738 (1995).

${ }^{37}$ M. Buttiker, in Quantum Dynamics of Submicron Structures, edited by H.A. Cerdeira (Kluwer, Dordrecht, 1995), p. 657.

${ }^{38}$ C.C. Wan, T.D. Jesus, and H. Guo, Phys. Rev. B 57, 11907 (1998).
${ }^{39}$ G. Taraschi et al., Phys. Rev. B 58, 13138 (1998).

${ }^{40}$ N.D. Lang and A.R. Williams, Phys. Rev. B 18, 616 (1978).

${ }^{41}$ The frequency of 0.0006 a.u. corresponds to $4 \times 10^{12} \mathrm{~Hz}$.

${ }^{42}$ J. Wang, Y.J. Wang, and H. Guo, Appl. Phys. Lett. 65, 1793 (1994). 